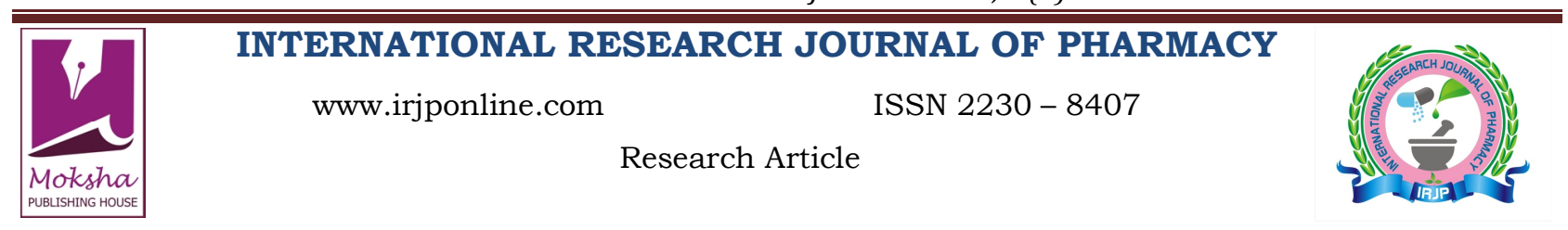

\title{
NINETY PERCENT DRUG UTILIZATION IN PATIENTS OF UPPER RESPIRATORY TRACT INFECTION
}

Lalit Patil, Avinash Khairnar*

University Department of Interpathy Research and Technology, Maharashtra University of Health Sciences, Nashik, India

*Corresponding Author Email: avinashkhairnar@gmail.com

Article Received on: 13/03/13 Revised on: 01/04/13 Approved for publication: 17/05/13

DOI: $10.7897 / 2230-8407.04642$

IRJP is an official publication of Moksha Publishing House. Website: www.mokshaph.com

(C) All rights reserved.

\section{ABSTRACT}

DU studies include descriptive epidemiological approaches to the study of drug utilization, but also the assessment of how drug utilization relates to the effects of drug use, beneficial or adverse. ${ }^{28}$ Therefore, present DU study was undertaken to analyze the prescribing patterns in the URTI. This was an observational, prospective, cross sectional study. Total 200 subjects and their prescriptions were studied in which 100 subjects were from ENT department of civil hospital, Nashik, India and 100 were from private ENT hospital, Nashik, India. Drug Utilization Form was filled with the help of Prescribed Drugs and Interview of subjects. Study core indicators were studied. DU 90\% was achieved with Amoxicillin, Azithromycin, Sulfamethoxazole + Trimethoprim and Cefixime which were the most prescribed drugs with having percentage of prescribing $52.5 \%, 22.5 \%, 7.5 \%, 6 \%$ respectively. The average number of drugs per prescription was 03 . Present study showed that percentage of prescriptions with an antibiotic prescribed was $91.5 \%$. Percentage of drugs prescribed from essential drug list was $56.75 \%$. Drug Utilization study in URTI showed for treatment of URTI antibiotics were used as first line of treatment but it should be get used as per guidelines and as per indication. Patient care indicators and Prescribing indicators should be given the importance. Overall, awareness among the physicians should be get increased by doing Drug Utilization studies among other diseases also to make health care society more responsible and patient caring.

Keywords: Drug Utilization study, DU 90\%, Prescribing Pattern, Observational, URTI.

\section{INTRODUCTION}

The Upper Respiratory Tract (URT) includes nose, the para nasal sinuses, adenoids, tonsils, nasopharynx and eustachian tube. ${ }^{18}$ The infections of these-URTI are common in community. More than 200 viruses can cause URTI. ${ }^{1}$ Acute respiratory infection accounts for $20-40 \%$ of outpatient and $12-35 \%$ of inpatient attendance in a General Hospital. Common cold does not require Anti Microbial Agent (AMA) unless it is complicated by acute otitis media with effusion, tonsillitis, sinusitis and lower respiratory tract infection. ${ }^{9}$ The drugs used for the URTI are antibiotics like Amoxicillin, Amoxicillin + Clavulinic acid, Cefixime, Cefuroxime ${ }^{25}$, common constituents of such medication include first generation antihistamines, antipyretics or anti-inflammatory agents (Ibuprofen), cough suppressants such as dextromethorphan, expectorants (Guaifenesin) and decongestants such as pseudoephedrine and phenylpropanolamine. ${ }^{17}$ Antibiotic treatment is beneficial to children only if symptoms persist for 10-14days without improvement. In present scenario antibiotic is a highly misused medicine. ${ }^{5}$ This is despite large amounts of evidence on the self-limiting nature of acute URTI. Reasons for inappropriate antibiotic use range from a patient's perception that antibiotics are beneficial in URTI, to the doctor's attempt to secure the patient's satisfaction with the consult by meeting such expectations. ${ }^{23}$ The World Health Organization (WHO) addressed DU as the "marketing, distribution, prescription and use of drugs in a society, considering its consequences, medical, social, and economic". DU research and pharmacoepidemiology may provide insights into the various aspects of drug use and drug prescribing such as pattern of use, quality of use, determinants of use, and outcomes of use. ${ }^{27}$ Rational drug use demands access to unbiased information. New drugs and new modes of therapy are also steadily being introduced. The quality of medical care requires prescribing to be judicious, appropriate, safe, effective and economic. "Good" prescribing is a complex balance between various conflicting factors. ${ }^{12}$ Drug use is a complex process. In any country a large number of socio-cultural factors contribute to the ways drugs are used. In India, these include national drug policy, illiteracy, poverty, use of multiple health care systems, drug advertising and promotion, sale of prescription drugs without prescription, competition in the medical and pharmaceutical market place and limited availability of independent, unbiased drug information. The complexity of drug use means that optimal benefits of drug therapy in patient care may not be achieved because of underuse, overuse or misuse of drugs. Inappropriate drug use may also lead to increased cost of medical care, antimicrobial resistance, adverse effects and patient mortality. ${ }^{6}$ The ultimate goal of drug utilization research is to assess whether drug therapy is rational or not. To reach this goal, methods for auditing drug therapy towards rationality are necessary. ${ }^{2}$ DU studies include descriptive epidemiological approaches to the study of drug utilization, but also the assessment of how drug utilization relates to the effects of drug use, beneficial or adverse. ${ }^{27}$ It is essential to study DU in patients of URTI because of wide prevalence of URTI and use of antibiotics and other group of drugs for URTI is increasing day-by-day. Studying DU in treatment of URTI helps to develop policy for the society to reduce the resistance of antibiotics. DU study in URTI gives exact scenario of current therapies and help to suggest improvements for maintaining the health of society. DU $90 \%$ is ideal tool for studying drug utilization. Therefore, present DU study was undertaken to analyze the prescribing patterns in the URTI. The aim of study was to evaluate the Drug Utilization $90 \%$ in patients of upper respiratory tract infection in OPD and IPD of ENT Department of Civil Hospital Nasik, India and Private ENT hospital, Nashik, India.

\section{MATERIALS AND METHODS}

Study was conducted in compliance with the protocol, ICHGCP, ICMR, Schedule ' $Y$ ' guidelines and Indian regulatory requirements. This was an observational, prospective, cross sectional study. This study was carried out in Civil and Private ENT hospitals of Nasik, India. Approval from Institutional Ethics Committee was taken prior to initiation of 
the study. Signed dated written informed consent was taken from all subjects after providing them with patient information sheet and informed consent form before screening. Total 200 subjects and their prescriptions in which 100 subjects were from ENT department of civil hospital, Nashik, India and 100 were from private hospital, Nashik, India who was prescribed for URTI fulfilling inclusion criteria was selected for the study. Screening of subjects was done according to inclusion and exclusion criteria with help of prescreening form and subjects were enrolled in study. Patient's prescription was studied for DU in accordance with protocol. Patient's prescription was studied as well as Drug Utilization Form was filled with the help of Prescribed Drugs and Interview of subjects. For assessment and evaluation of antibiotics resistance, patients prescribed for URTI were divided in two groups as patients prescribed with antibiotics and patients prescribed other than antibiotics. Telephonic follow-up was taken of patients of both the groups (maximum up to 7days). Patients were asked for days required for relief of symptoms of URTI and were assessed with given duration of action of drug (either antibiotics or other group of drugs). Resistance to antibiotics was evaluated with three categories drugs having no effect on relief of symptoms, drugs having slow duration of action on relief of symptoms and drugs acting as per given duration of action for relief of symptoms. Data was recorded on follow-up form. Core indicators were studied. ADR if found (syncope, allergic reaction, leg and ankle edema etc.) was recorded in ADR form.

\section{Study Duration: 10weeks}

\section{Primary Objective}

- To determine and calculate DU $90 \%$ in patients of upper respiratory tract infection by studying DUF of patients of URTI.

- To obtain data for prescribed drugs, drugs prescribing patterns, the duration of treatment in patients of URTI with the help of DUF and interview of patients of URTI.

- To calculate the percentage of drugs which are prescribed by their generic names and percentage of Encounters with medicine which are prescribed in patients of URTI by studying DUF of patient of URTI.

\section{Secondary Objective}

- To calculate the cost of treatment prescribed for URTI.

- To evaluate the effect, impact of Drugs Utilization Study on individual, society for their health in patients of URTI by accessing result of study.

- Assessment and evaluation of antibiotics resistance with help of telephonic follow up of patients of URTI and follow up form.

\section{Inclusion Criteria}

- The patients diagnosed and prescribed for URTI were included in the study.

- Patients having age group in between 18 years to 60years.

- Patient with either sex.

- Patient willing to give voluntarily free written informed consent.

\section{Exclusion Criteria}

- Patient admitted in hospital for any chronic disease.
- Patient having previous $\mathrm{h} / \mathrm{o}$ Myocardial Infarction, Stroke, Congestive Cardiac Failure in past six months.

- Immunocompromised patients.

- Pregnant and nursing women.

- Patient not willing to give written informed consent.

\section{Core Indicators}

\section{Prescribing indicators}

a) Average number of drugs per encounter

b) Percentage of drugs prescribed by generic name

c) Percentage of encounters with an antibiotic prescribed

d) Percentage of encounters with an injection prescribed

e) Percentage of drugs prescribed from essential drug list

\section{Patient Care Indicators}

a) Average Consulting Time

b) Average dispensing time

c) Percentage of drugs actually dispensed

d) Patients' knowledge of correct dosage

\section{RESULTS}

The total 200 (100 from civil hospital and 100 from private hospital) patients according to selection criteria were selected for present study. Total number of prescriptions which were analyzed was 200 and total number of drugs prescribed was 629 in which 349 drugs were prescribed from civil hospital and 280 drugs were prescribed from private hospital. From collected data for Drug utilization pattern for treatment of URTI in civil hospital and private hospital. DU 90\% was achieved. Table 1 is showing DDD and Drug Utilization 90\% in patients of URTI. Table 1 shows percentage of drugs used in the prescriptions given to patients of URTI in both Civil and Private ENT hospital. With this percentage DU $90 \%$ was achieved for utilization of drugs in URTI. Table 2 shows the details of prescribing indicators in the prescriptions of patients of URTI in both Civil and Private Hospital. Table 3 shows the results of core indicators which were related to patient care. On the basis of the values given in Table 3, the average number of drugs per prescription was 03 . Percentage of drugs prescribed by Generic name was $45 \%$. Present study showed that percentage of prescriptions with an antibiotic prescribed was $91.5 \%$. Percentage of drugs prescribed from essential drug list was $56.75 \%$. Average consulting time was 2 minutes 50 seconds. Average dispensing time was 5 minutes 50 seconds. Percentage of drugs actually prescribed was $100 \%$. Percentage of patient showing knowledge about correct dosage was $77.5 \%$. Table 4 gives the results for percentage of drugs prescribed by physician in Civil Hospital to patients of URTI. It shows the number of antibiotics routinely prescribed for URTI in civil hospital. Table 5 gives the results for percentage of drugs prescribed by physician in Private ENT Hospital to patients of URTI. It shows the number of antibiotics routinely prescribed for URTI in Private ENT hospital. Table 6 shows number of drugs prescribed with their generic name in both Civil and Private Hospital for URTI. Table 7 shows average cost of therapy per day in civil hospital and in Private hospital. With these values the average cost of therapy during study found was 18.90/per day. Table 8 shows the data obtained by taking telephonic follow up in patients of URTI both in Civil as well as Private hospital. This observation suggests that, resistance may get develop due to irrational and wide use of antibiotics in those $17 \%$ patients of civil hospital. Graph 1 shows the data for prescribing indicators observed during drug utilization study for URTI. 
Table 1: Percentage of Drugs prescribed for Drug Utilization used in URTI

\begin{tabular}{|c|c|c|c|c|}
\hline Drugs Used & ATC CODE & DDD & Percentage of Drug Used (\%) & DU 90 \% \\
\hline Amoxicillin & J01CA04 & $1 \mathrm{~g}$ & 52.5 & 52.5 \\
\hline Azithromycin & J01FA10 & $0.3 \mathrm{~g}$ & 22.5 & 75 \\
\hline Sulfamethoxazole+Trimethoprim & J01EE01 & $1.92 \mathrm{~g}$ & 7.5 & 82.5 \\
\hline Cefixime & JO1DD08 & $0.4 \mathrm{~g}$ & 6 & 88.5 \\
\hline Ciprofloxacin & J01MA02 & $1 \mathrm{~g}$ & 3 & \\
\hline
\end{tabular}

DU $90 \%$ was achieved with drugs 1-5

Table 2: Details for Prescribing Indicators in patient's prescriptions

\begin{tabular}{|c|c|c|}
\hline Indicator & Civil Hospital & Private Hospital \\
\hline Number of drugs prescribed & 349 & 280 \\
\hline Drugs prescribed with generic name & 283 & 0 \\
\hline Number of antibiotic prescriptions & 93 & 90 \\
\hline Number of drugs prescribed from essential drug list & 247 & 110 \\
\hline
\end{tabular}

Table 3: Details of Patient Care Indicators in Patients Prescriptions

\begin{tabular}{|c|c|c|}
\hline Indicator & Civil Hospital & Private Hospital \\
\hline Average Consulting time & 1 minute & 4 minutes \\
\hline Average Dispensing time & 5 minutes & 6 minutes \\
\hline Number of antibiotic prescriptions & 93 & 90 \\
\hline Number of patients showing awareness about prescribed drugs & 37 & 95 \\
\hline Number of patients showing Compliance about prescribed drugs & 80 & 98 \\
\hline
\end{tabular}

Table 4: Antibiotic agents prescribed for treatment of URTI in Civil hospital

\begin{tabular}{|c|c|}
\hline Antibiotic agent & Number of prescriptions (\%) \\
\hline Amoxicillin & 67 \\
\hline Azithromycin & 20 \\
\hline Ciprofloxacin & 06 \\
\hline
\end{tabular}

Table 5: Antibiotic agents get prescribed for treatment of URTI in Private ENT Hospital

\begin{tabular}{|c|c|}
\hline Antibiotic agent & Number of prescriptions (\%) \\
\hline Amoxicillin & 38 \\
\hline Azithromycin & 25 \\
\hline Cefixime & 12 \\
\hline Septran (Sulfamethoxazole + Trimethoprim) & 15 \\
\hline
\end{tabular}

Table 6: Drugs prescribed with their generic name

\begin{tabular}{|c|c|c|}
\hline Description & Civil Hospital & Private ENT Hospital \\
\hline Number of Drugs prescribed with their Generic Name & 283 & 0 \\
\hline
\end{tabular}

Table 7: Average Cost of Therapy for URTI in Civil and Private Hospital

\begin{tabular}{|c|c|c|}
\hline Description & Civil Hospital (Rs) & Private ENT Hospital (Rs) \\
\hline Average cost of therapy per day & $3.60 /-$ & $34.20 /-$ \\
\hline
\end{tabular}

Table 8: Details of Telephonic Follow-Up Of Patients

\begin{tabular}{|c|c|c|}
\hline Description & Civil Hospital (\%) & Private Hospital (\%) \\
\hline Percentage of patients got relief from symptoms as per given duration of action for prescribed drugs & 73 & 99 \\
\hline $\begin{array}{c}\text { Percentage of patients showed slow action for prescribed drug from symptoms compare to given } \\
\text { duration of action of prescribed drugs }\end{array}$ & 3 & 0 \\
\hline $\begin{array}{l}\text { Percentage of patients showed no action for prescribed drug from symptoms compare to given } \\
\text { duration of action of prescribed drugs }\end{array}$ & 17 & - \\
\hline
\end{tabular}




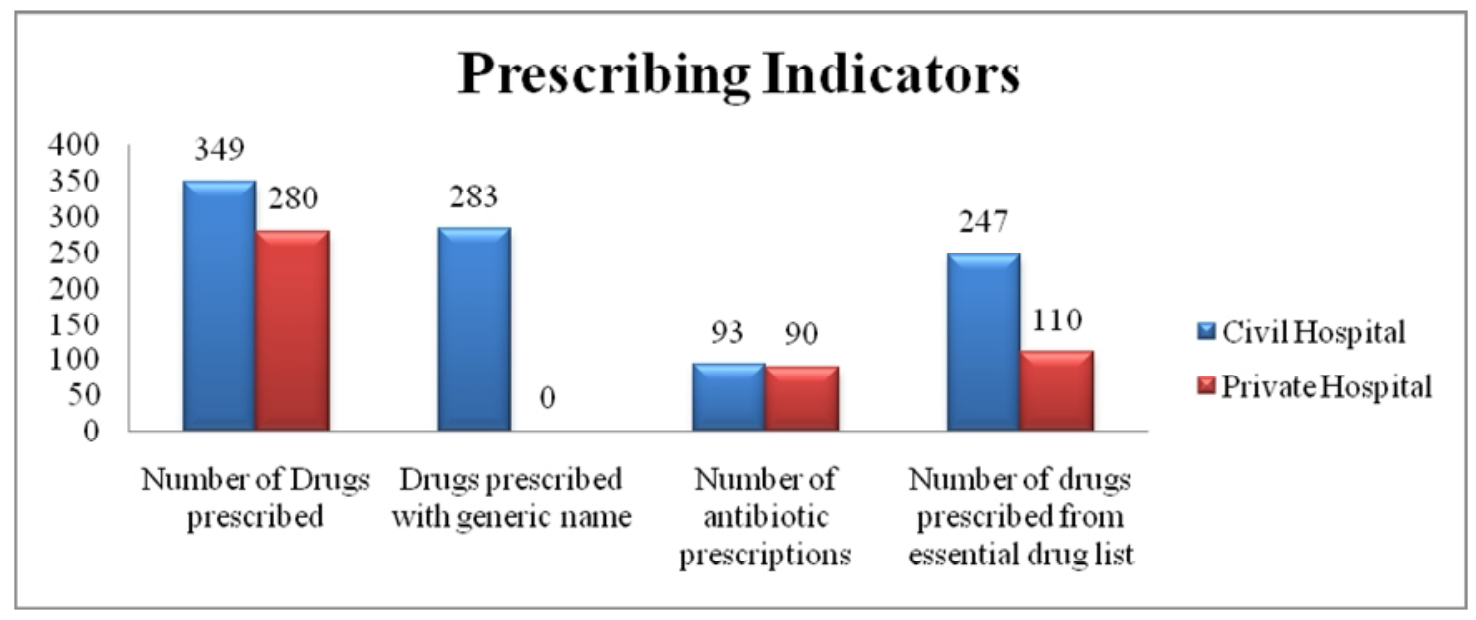

Graph 1: Details of Prescribing Indicators in patient's prescriptions

\section{DISCUSSION}

Drugs play an important role in improving human health and in promoting well being. To produce the desired effect, they have to be safe and efficacious and have to be used rationally. The irrational use of drugs is a common occurrence throughout the world. Drug prescriptions form a very important point of contact between the doctors and the patients. Amoxicillin was prescribed as a first preference in treatment of URTI as having the percentage $52.5 \%$. Azithromycin was used by $22.5 \%$. Sulfamethoxazole + Trimethoprim, Cefixime were used $7.5 \%$ and $6 \%$ respectively. As the percentage showed Amoxicillin was used as first line of treatment for URTI. This shows number of drugs routinely prescribed for URTI. Still conventionally Amoxicillin is used as a first line of antibiotic for the treatment of URTI. Percentage of Amoxicillin and Azithromycin is much higher than other drugs prescribed for URTI. 349 drugs were prescribed in civil hospital and 280 drugs were prescribed in private hospital for URTI. More drugs were used in civil hospital for treatment as compared to private hospital. Out of drugs prescribed in civil hospital 283 drugs were prescribed by generic name means almost $81.08 \%$ drugs were prescribed with generic name. In private hospital no prescription was having drugs prescribed with their generic name. In civil hospital 93 prescriptions were having antibiotics prescribed in the prescription. In private hospital 90 prescriptions were prescribed with antibiotics for treatment of URTI. In civil hospital prescriptions were having more number of drugs prescribed from essential drug list as number was 247 compared to private hospital prescribed 110 drugs from essential medicine list. This results show that number of drugs prescribed for URTI in civil hospital is more than drugs prescribed in private hospital. Generic medicines are used in very less percentage in private hospital as compared medicines used in civil hospital. Prescriptions having antibiotics prescribed are used in both the hospitals nearly the same but use of antibiotic in private hospital is more accurate for the indication of drug as compared to antibiotics used in civil hospital. Average consultation time in Civil Hospital was 1 minute and in private hospital average consultation time was 4minutes. Average dispensing time in civil and private hospital was 4 minutes and 5minutes respectively. In civil hospital consultation time was very less because of number of patients were more and time provided for OPD was having limitations. On the other hand in private hospital number of patient was given sufficient time history taking, clinical findings and examination. In civil hospital $37 \%$ patients were aware about the prescribed medication, $80 \%$ were having compliance with prescribed drugs. As the patients coming to civil hospital were mostly uneducated henceforth percentage was low as compared to private hospital patients were well educated and having awareness and compliance with medication were $95 \%$ and $98 \%$ respectively. Amoxicillin, Azithromycin, Ciprofloxacin were antibiotics agents prescribed for URTI having percentage of prescription 67\%, 20\%,6\% respectively. Mostly Amoxicillin was used as first line antibiotic agent in treatment URTI. Antibiotics prescription was mostly dependant on availability of medicine. Table 5 showed antibiotics agents prescribed in prescriptions at private hospital. In private hospital antibiotics were prescribed as per indication henceforth various agents were used. Figure showed that same as civil hospital Amoxicillin was used as first line of treatment of URTI in private hospital but the percentage used was less as having $38 \%$. Azithromycin, Cefixime, Septran (Sulfamethoxazole + Trimethoprim) were used $25 \%, 12 \%, 15 \%$ respectively. In private hospital various antibiotics agents were used as compared to civil hospital. Use of antibiotics in private hospital is majorly according to indication of drug as compare to civil hospital. The reason behind this might be the availability of drugs in civil hospital is less than in private hospital. During the study we found the average Cost of therapy per day in civil hospital was 3.60/- and in private hospital was 34.20/- therefore average cost of therapy during study found was 18.90/-per day. Cost of therapy in private hospital was higher than civil hospital due to consultation charges, cost of medicine were higher than civil hospital. In this study, prescribing patterns of civil hospital showed very much irrationality as major percentage of prescriptions were get prescribed by internee students. Interns were prescribing the prescriptions without having proper knowledge of prescribed drugs about dose, duration, frequency, mechanism of action etc. Most of prescriptions were (Nearly 80-85\%) were get prescribed without knowing the drug indication and therefore drugs get prescribed irrelevant to symptoms of patients, mostly antibiotics were get prescribed very irrationally. $7 \%$ of prescriptions of civil hospital get prescribed only with multivitamin and antacid agents for the treatment of URTI. On other hand private hospital showed rational prescribing pattern with proper examination as well 
as consultation of patients and having knowledge of drug action, duration, frequency, dose etc. Drugs were prescribed as per indicated symptoms and with proper anti cold agent, anti cough agent wherever necessary. Antibiotics were prescribed as per symptoms of patients with proper combination for the treatment of URTI. Present study showed that there is need for improvement in prescription writing in civil hospital as majority of prescriptions were prescribed without proper formulation, dose, duration. There is a need to conduct similar studies in other departments of hospital, as well to audit a large number of prescriptions and to impart education to the prescriber on rational drug therapy for the benefits and for the safety of the patients.

\section{CONCLUSION}

Drug utilization study in URTI showed that for the treatment of URTI antibiotics were used as first line of treatment but it should be get used as per guidelines and as per indication. It will help to reduce the resistance to antibiotics. Patient care indicators and Prescribing indicators should be given the importance mostly in civil hospital during treatment of disease. In private hospital physician should take care of cost of therapy for treatment. Overall, awareness among the physicians should be get increased by doing Drug utilization studies among other diseases also to make health care society more responsible and patient caring.

\section{REFERENCES}

1. Balkhy $\mathrm{HH}$, Memish ZA, Bafaqeer S, Almuneef MA. Influenza a common viral infection among Hajj pilgrims: time for routine surveillance and vaccination. J Travel Med 2004; 11(2): 82-6. http://dx.doi.org/10.2310/7060.2004.17027 PMid:15109471

2. Bergman U, et al. The measurement of drug consumption. Drugs for diabetes in Northern Ireland, Norway and Sweden. European Journal of Clinical Pharmacology 1975; 8: 83. http://dx.doi.org/10.1007/ BF00561555 PMid:1233211

3. Bert Spilkar. Text book of Quality of Life and Pharmaco economics in Clinical Trial, $2^{\text {nd }}$ ed, Lippin Cott- Raven Publisher (Philadelphia) USA; 1996.

4. Dick E Zoutman, B Douglas Ford, Assil R Bassili. Antibiotic Prescribing By Family Physicians for Upper Respiratory Tract Infections, Int J Infect Control 2006; 2: 1.

5. Chaurasia Chandra Dr Rakesh- Medication errors in children. IAP Bulletin Academy today 2006; 2(3): 28-29.

6. Einarson T. Pharmco epidemiology. In: Parthasarathi G, Hansen KN, Nahata MC, editors. A Text book of Clinical Pharmacy Practice essential concepts and skills. $1^{\text {st }}$ ed., Hyderabad: Universities Press (India) Limited; 2008. p. 405-23

7. Gonzales R, Bartlett JG, Besser RE, et al. Principles of appropriate antibiotic use for treatment of acute respiratory tract infections in adults: background, specific aims, and methods, Ann Emerg Med 2001; 37: 690-697.http://dx.doi.org/10.1067/mem.2001.em376698 PMid: 11385342

8. Huug J van Duijn, Marijke M Kuyvenhoven, Hanneke M Tiebosch, François G Schellevis and Theo JM Verheij. Diagnostic labelling as determinant of antibiotic prescribing for acute respiratory tract episodes in general practice, BMC Family Practice; 2007.

9. Jain N, Lodha R, Kabra SK- Upper respiratory tract infections -IJP 2001; 68(12): 1135-8.

10. Johanna Nokso-Koivisto. Viral upper respiratory tract infections in young children, National Public Health Institute Mannerheimintie 166 FIN-00300 HELSINKI; 2004.
11. Kuehnert MD David A, Talan MD. ID NET Study Group, Antibiotic Use for Emergency Department Patients with Upper Respiratory Infections: Prescribing Practices, Patient Expectations and Patient Satisfaction, American College of Emergency Physicians. Published by Elsevier Inc 2007; 50(3): 213-220.

12. Martin RM. When to use a new drug. Aust Prescr 1998; 21: 67-68.

13. Meena K Nandimath, Sam Ahuja. Drug Prescribing Pattern in upper respiratory tract infection in children aged 1-14years; 2012.

14. Michi Sakai, Takuro Shimbo, Kazumi Omata, Yoshimitsu Takahashi, Kazunari Satomura, Tetsuhisa Kitamura, Takashi Kawamura, Hisamitsu Baba, Masaharu Yoshihara, Hiroshi Itoh. Cost-effectiveness of gargling for the prevention of upper respiratory tract infections, BMC Health Services Research 2008; 8: 258. http://dx.doi.org/10.1186/1472-6963-8258 PMid:19087312 PMCid:2651874

15. Mika J Makela, Tuomo Puhakka, Olli Ruuskanen, Majja Leinomen, Pekka Saikku, Marko Kimpimaki, Solile Blomqvist, Timo Hyypia and Pertti Arstila. J Clin Microbiol. Viruses and Bacteria in the Etiology of common Cld, 1998; 36(2).

16. Mireya Aznar, Raul Mejia, Robert Wigton, Roberto Fayanas. Predictors of Antibiotic Prescription In Respiratory Tract Infections By Ambulatory Care Practitioners, Medicina (Buenos Aires) 2005; 65: 501506.

17. MMWR. Infant deaths associated with cough and cold medications- two states, 2005; 2007; 12, 2007.

18. Richard E Behrman, Robert M, KliehmanMD, HalB Jenson MD. Infection of Upper Respiratory Tract. Nelson textbook of Pediatrics WB Saunders Company; 2000. p. 1261

19. NICE clinical guideline 69 Developed by the Centre for Clinical Practice at NICE. Respiratory tract infections- antibiotic prescribing of antibiotics for self-limiting respiratory tract infections in adults and children in primary care, CG069; 2008.

20. Nick Dunn, Dan Lane, Hazel Everitt and Paul Little. Use of antibiotics for sore throat and incidence of quinsy, British Journal of General Practice 2007; 57: 45-49. PMid:17244424 PMCid:2032700

21. Petersen I, Johnson AM, Duckworth G et al. Protective effect of antibiotics against serious complications of common respiratory tract infections: retrospective cohort study with the UK General Practice Research Database. BMJ 2007; 335: 982. http://dx.doi.org/10.1136 /bmj.39345.405243.BE PMid:17947744 PMCid:2072032

22. Richelle J Cooper, MD MSHS, Jerome R Hoffman MA, MD, John G Bartlett MD, Richard E Besser MD, Ralph Gonzales MD, MSPH, John M Hickner MD, MSc and Merle A Sande MD. Principles of Appropriate Antibiotic Use for Acute Pharyngitis Adults: Background, Ann Intern Med 2001; 134: 509-517.

23. Scott JG, Cohen D, Dicicco-Bloom B, et al. Antibiotic use in acute respiratory infections and the ways patients pressure physicians for a prescription. J Fam Pract 2001; 50: 853-8. Erratum in: J Fam Pract 2001; 50: 1077. PMid:11674887

24. Shaun Mohan, Kavita Dharamraj, Ria Dindial, Deepti Mathur, Vishala Parmasad, Joseph Ramdhanie, Jason Matthew and Lexley M Pinto Pereira. Physician behaviour for antimicrobial prescribing for pediatric upper respiratory tract infections: a survey in general practice in Trinidad, West Indies, Annals of Clinical Microbiology and Antimicrobials 2004; 3: 11. http://dx.doi.org/10.1186/1476-0711-3-11 PMid:15196306 PMCid:441403

25. Ramchandra K, Narendranath Sanji, HS Somashekhar, Abhishek Acharya, Keerti Sager J. Trends in prescribing antimicrobials in tertiary hospital for upper respiratory tract infection, Int J Pharmacol and Cli SCi 2012; 1: 15, 18 .

26. Tan YSL, Hong CY, Chong PN, Tan ESL, Lew YJ, Lin RTP. Knowledge that upper respiratory tract infection resolves on its own is associated with more appropriate health-seeking behavior and antibiotic cognition, Med J 2006; 47(6): 518.

27. WHO booklet "Introduction to Drug Utilization Research" ISBN 924 $156234 \mathrm{X} ; 2003$.

Cite this article as:

Lalit Patil, Avinash Khairnar. Ninety percent drug utilization in patients of Upper respiratory tract infection. Int. Res. J. Pharm. 2013; 4(6):189-193 